\title{
EL RESPETO A LA IDENTIDAD COMO FUNDAMENTO DE LA EDUCACIÓN INTERCULTURAL
}

\author{
Respect for identity as a basis for intercultural education
}

\section{Le respect de l'identité en tant que base de l'éducation interculturelle}

David MERINO MATA

Universidad de Málaga. Facultad de Ciencias de la Educación. Departamento de Teoría e Historia de la Educación. Campus de Teatinos, s/n. 29071 Málaga. Correo-e:dmerino@uma.es

Fecha de recepción: marzo de 2004

Fecha de aceptación definitiva: octubre de 2004

BIBLID [(1130-3743) 16, 2004, 49-64]

RESUMEN

En este artículo se ofrece una reflexión acerca de la importancia del respeto a la identidad a la hora de establecer relaciones interculturales, y por tanto de cara a la aplicación de programas para favorecer el desarrollo de la educación intercultural. Se pone de relieve la necesidad de atender al individuo, es decir, de respetar y valorar su identidad personal como prerrequisito para el posterior establecimiento de vínculos interculturales que nos lleven al mutuo respeto y a la comunicación intercultural.

Palabras clave: identidad individual, identidad colectiva, educación intercultural.

SUMMARY

This article offers a reflection of the importance of respect for identity when establishing intercultural relations and therefore, in the application of programmes to aid the development of intercultural relations. It highlights the need for paying attention to the individual, i.e. respecting and valuing personal identity as a prerequisite for the 
subsequent establishment of intercultural links which lead us to mutual respect and intercultural communication.

Key words: individual identity, collective identity, intercultural education.

SOMMAIRE

Cet article offre une réflexion sur l'importance du respect de l'identité lorsque s'établissent des rapports interculturels, et par là-même celle de l'application de programmes pour favoriser le développement de l'éducation interculturelle. L'accent est mis sur le besoin de prêter attention à l'individu, c'est-à-dire de respecter et d'apprécier son identité personnelle comme démarche préalable pour établir par la suite des liens interculturels, qui aboutissent au respect mutuel et à la communication interculturelle.

Mots clef: identité individuelle, identité collective, éducation interculturelle.

\section{INTRODUCCIÓN}

El fenómeno de la interculturalidad se ha convertido en una cuestión de máximo interés para un gran número de profesionales dedicados a distintos campos de estudio. Basta con revisar la literatura sobre el tema para observar cómo la producción bibliográfica se ha ido multiplicando progresivamente a un ritmo vertiginoso: estudios sobre inmigración, sobre la situación de minorías étnicas en distintos países, sobre los derechos de estas minorías, sobre los programas para favorecer la inserción educativa y social de dichos grupos, y un largo etcétera de temáticas que ponen de manifiesto la creciente importancia que este hecho despierta tanto a nivel nacional como internacional.

Por su parte, la educación intercultural ha adquirido la consideración de herramienta fundamental de cara a superar los prejuicios que existen hacia los grupos minoritarios, así como para conseguir una interacción cultural en el contexto educativo basada en el mutuo enriquecimiento que para el alumnado puede implicar esa coexistencia.

Todo ello ha convertido el tema de la identidad en el centro de numerosos análisis que, como no podía ser de otro modo, han impregnado la reflexión y la acción educativas. En esa dirección camina el siguiente artículo, en el que intentamos evidenciar la importancia y la necesidad de centrar los procesos de educación intercultural en el respeto a la identidad de los educandos, comenzado por la aceptación individual como fundamento del reconocimiento cultural. En este sentido, si somos capaces de aceptar y respetar al otro por quien es - persona-, estaremos favoreciendo su libertad de elección a la hora de construir una identidad dinámica que otorgue sentido a la idea de enriquecimiento mutuo que debe nutrir los procesos interculturales. 


\section{LA IDENTIDAD COMO EJE DEL DEBATE SOBRE EL INTERCULTURALISMO}

El tema de la identidad es básico para poder tratar la cuestión de las relaciones interculturales, ya que cada grupo cultural posee unas características propias que conforman su identidad. Sin embargo, en todos los grupos hay personas que, en muchos casos, han tenido que realizar un esfuerzo para que ciertas dimensiones de sus identidades personales coincidan, otorgando al grupo una idiosincrasia que por una parte les identifica como grupo, y por otro lado les confiere singularidad frente a las demás colectividades.

Un principio general que debe guiar toda educación intercultural es la necesidad de que ésta abarque a todo el alumnado y no solamente a aquellos alumnos pertenecientes a culturas minoritarias, es decir, "la educación intercultural debe ser un proyecto cósmico, con acciones y actuaciones dirigidas a la totalidad de la población" (Froufe, 1999, 16). Por tanto, la educación intercultural es, en este sentido, una educación pluralista, educación para la igualdad y para el respeto, por lo que la cuestión de la identidad se convierte en un tema central a la hora de reflexionar sobre la atención educativa en contextos de diversidad cultural. En este sentido podemos plantearnos múltiples cuestiones como: "ipueden coexistir positivamente identidades que difieran notablemente en un mismo espacio?", "¿el mantenimiento de una identidad colectiva implica necesariamente una uniformidad de identidades individuales?", o "ipuede una persona encerrarse en su propia identidad aun en el caso en que ésta no tenga un mínimo de puntos en común con el grupo social en el que se desenvuelve?". En cualquier caso es muy habitual olvidar que todos los grupos están formados por personas con características, trayectorias vitales o líneas de pensamiento propias, que pueden compartir o no con el resto del grupo en el que las encuadramos. Sin embargo, tendemos a agruparlas y por tanto a homogeneizarlas con el colectivo correspondiente, olvidando de esta manera la individualidad, la "mismidad" que le es consustancial a cada persona y por tanto nos centramos en la aplicación de recetas con el convencimiento de que pueden servir para todos los miembros de un grupo concreto. Como exponen los profesores Jordán, Ortega y Mínguez:

Ha habido, hasta ahora, una excesiva escolarización de la educación intercultural, acentuando los aspectos cognitivos de la misma como si sólo, o principalmente, se tratara de conocer y respetar las ideas, creencias, tradiciones y lengua de una comunidad, en una palabra, la cultura del otro, haciendo abstracción o relegando a un segundo plano al sujeto concreto que está detrás de esa cultura $(2002,114)$.

\section{3. ¿QUIÉN SOY YO?: LA IDENTIDAD INDIVIDUAL}

"La identidad es una necesidad básica del ser humano. Es tan necesaria como el afecto o el alimento" (Castillo, 2004, 379). Efectivamente, la identidad es una característica exclusivamente humana, uno de los factores fundamentales que diferencian al hombre de todas las demás criaturas. La identidad personal no es algo 
meramente innato, sino que su formación, consolidación y evolución están directamente relacionadas con factores sociales, culturales, emocionales, familiares, históricos, etc.

Según Erikson (1989), la identidad individual es una definición de uno mismo que en parte está implícita, y que una persona adulta elabora como el elemento constituyente de su personalidad, y esta definición se sigue construyendo a lo largo de toda la vida. Esta conceptualización del término identidad individual nos aporta algunas de sus características fundamentales, es decir, su dinamismo y su posibilidad de cambio y por tanto de adaptación. Tal como afirma Maalouf $(1999,35)$ «la identidad no se nos da de una vez por todas, sino que se va construyendo y transformando a lo largo de toda nuestra existencia", o dicho de otro modo, "la identidad se forma y se transforma continuamente con relación a las formas por las que somos representados y tratados en los sistemas culturales que nos circundan, dando la posibilidad a que en nosotros coexistan identidades contradictorias, de modo que nuestras identificaciones cambian incesantemente" (Soriano, 2004, 207).

El individuo, al modificar e incorporar elementos nuevos a dicha identidad personal tiene la capacidad de adaptarse a contextos donde las identidades externas difieran de la suya propia. A pesar de ello, los sujetos tienden a defender su propia identidad personal, a la vez que necesitan compartir dicha identidad con los miembros de su propio grupo cultural, social, religioso político, etc., conformando lo que denominamos identidades colectivas.

Para Muñoz Sedano (1997), la identidad personal es el sentido del yo que proporciona una unidad a la personalidad en el transcurso del tiempo, el reconocimiento de que mi yo, que vivió, participó y tomó decisiones en tiempos y lugares diversos a los actuales, es sin embargo idéntico: el mismo yo del presente. Tal unidad en la personalidad implica una estabilidad de la propia identidad. Cuando la persona no tiene una identidad estable se siente al borde de la crisis, desarrolla sentimientos de inferioridad, su conducta se bloquea y es incapaz de funcionar con normalidad. De igual forma, todo individuo negocia su identidad con su entorno, por lo que se nos hace difícil tomar decisiones individuales que pudieran llevarnos a ser rechazados por el grupo. En caso de que se produzca tal rechazo, el sujeto busca otro grupo donde su identidad sea aceptada:

Toda identidad va cambiando y requiere de la alteridad. No se puede reconocer una identidad si a la vez no se reconoce una alteridad que se puede presentar como un antagonista. Estos antagonismos nos crean conflictos con los demás, pero también con nosotros mismos. Si el otro no confirma mi identidad, se transforma en una amenaza y es frecuente que se intente evitar el contacto con aquellos que nos amenazan, que ponen en riesgo mi identidad, mi autoimagen o mi autoestima (Castillo, 2004, 379-380).

Malewska-Peyre (1982) apuntaba la presencia de crisis de identidad en hijos de inmigrantes, en los que se hallaba una correlación significativa entre la vivencia del racismo y la imagen de sí mismos, es decir, dichos alumnos al comprobar que 
su origen cultural era motivo de rechazo desarrollaban sentimientos de inferioridad, ya que el grupo formado por otras personas configura el lugar donde el sujeto pone a prueba su autoestima, y la valoración que de él se tiene (Monreal, Muñoz y Martínez, 1990). Efectivamente, cuando el sujeto comprueba que su identidad no es aceptada por el grupo mayoritario, una opción lógica es la de reunirse en torno a aquellas personas que comparten un número significativo de rasgos identificatorios (segregación), ya que el mantenimiento de relaciones interétnicas positivas se ve imposibilitado. Tal rechazo puede traducirse en un deterioro del autoconcepto del sujeto. Según Muñoz Sedano (1997), las consecuencias de un autoconcepto minusvalorado sobre el rendimiento escolar y sobre la adaptación personal y social pueden ser muy graves, siendo uno de los aspectos más importantes a tener en cuenta en la educación de inmigrantes y minorías étnicas marginadas. En este sentido, un trabajo desarrollado en la Universidad de Málaga (Esteve, Merino, Rius, Canto y Ruiz, 2003) con una muestra de 1.886 alumnos de primer ciclo de Educación Secundaria Obligatoria, escolarizados en tres localidades de Campo de Gibraltar (La Línea de la Concepción, San Roque y Algeciras) y la Ciudad Autónoma de Melilla establece una clara relación entre el autoconcepto de dichos alumnos y la tendencia a recurrir a respuestas agresivas. Para dicho estudio empleamos la Escala de Autoconcepto de Piers-Harris y la Escala de Mediadores Cognitivos de la Conducta Agresiva, comprobándose que cuanto más bajo era el autoconcepto de los alumnos existían mayores posibilidades de que utilizaran conductas agresivas en su relación con los demás, algo que se repetía como una constante en los cinco grupos culturales estudiados ${ }^{1}$, es decir, nuestros datos sugieren que cuanto más rechazado se siente el alumno, cuanto peor se ve a sí mismo y peor imagen tiene de su conducta, tiende en mayor medida a justificar y a emplear respuestas agresivas.

Obsérvese por tanto que el deterioro del autoconcepto, derivado en este caso del rechazo, puede traducirse en toda una serie de conductas agresivas que, por otra parte, cada vez están más presentes en nuestros centros escolares. Esta afirmación está avalada por numerosas investigaciones (Schoenfeld, 1988; Long, 1990; Wiehe, 1991; Kirschner, 1992), así como por el estudio elaborado en la Universidad de Málaga del que venimos hablando, de lo cual se deduce que la elaboración de líneas de intervención para los profesores en contextos interculturales, atendiendo

1. Los.alumnos pertenecían a cinco grupos culturales diferentes:

1. Grupo de alumnos de cultura "mayoritaria-cristiana", compuesto por los alumnos de nacionalidad española de origen y de religión cristiana, entre ellos casi todos católicos, si bien existía un pequeño grupo de evangélicos y testigos de Jehová $(n=1.565)$.

2. Grupo de alumnos de cultura musulmana, donde fueron integrados los niños de origen magrebí y de religión musulmana con independencia de su nacionalidad $(n=245)$.

3. Grupo de alumnos de cultura "mixta hispano-musulmana", que estaba formado por los niños de familias en las que uno de sus padres pertenecía a uno de los dos grupos antes descritos $(n=21)$.

4. Grupo de alumnos de cultura hebrea $(n=16)$.

5. Grupo de alumnos de cultura gitana $(n=39)$. 
prioritariamente al autoconcepto de los alumnos, puede constituirse en una forma de prevención de situaciones potencialmente conflictivas (Esteve, Merino, Ruis, Canto y Ruiz, 2003).

Uno de los principales estudiosos sobre el tema de la identidad fue Erik Erikson, profesor de Desarrollo Humano en la Universidad de Harvard al que anteriormente hemos hecho referencia. Para este autor, la adquisición de la identidad se revela como la principal tarea de la adolescencia, implicando tres componentes: un sentido de unidad entre las autoconcepciones, un sentido de continuidad a través del tiempo de estos atributos propios y un sentido de la correspondencia mutua entre las concepciones que sostiene el individuo sobre sí mismo y aquellas que sobre él mantienen las personas significativas de su entorno (Erikson, 1989). Como podemos comprobar, Erikson hace referencia a la necesidad de reconocimiento, es decir, que para que mi identidad sea estable es necesario que sea reconocida por los demás, y, en cualquier caso, será preciso que ese reconocimiento externo concuerde con lo que yo pienso sobre mí mismo. Erikson sitúa el problema de la adquisición de la identidad en la quinta de sus etapas evolutivas, denominada “identidad frente a confusión de la identidad». Para él, la identidad del adolescente debe realizar un trabajo de integración de las vicisitudes de su libido, las aptitudes desarrolladas a partir de lo congénito y las oportunidades que se le ofrecen de adoptar diversos papeles sociales. Por lo tanto Erikson reconoce la importancia del medio social en la conformación de la identidad individual. Obviamente, el individuo se desarrolla inmerso en una sociedad, y necesita ser aceptado por el grupo. Para que esto suceda será básico que la identidad personal no entre en conflicto con la identidad del grupo, por lo que podemos establecer una clara relación entre identidad individual e identidades colectivas.

Para Charles Taylor $(1996,10)$ ala identidad individual define de alguna manera el horizonte de mi propio mundo moral». Esta tesis se justifica con la afirmación de que el individuo, a partir de las características de su propia identidad, establece una escala de valores en la que queda reflejado aquello que resulta más o menos importante para él. En definitiva, a partir de nuestra propia identidad nos vamos a permitir o a prohibir conductas y acciones; nuestra propia identidad va a resultar de esta forma esencial en nuestra propia visión del mundo.

Hemos de aclarar que para que la identidad sea sólida y estable, ésta debe haberse desarrollado en un contexto de libertad, ya que «la identidad es algo personal, potencialmente original e inédita y, por consiguiente, inventada o asumida en cierta medida" (Taylor, 1996, 12). Efectivamente, elaborar la propia identidad es un privilegio que sólo ejercen quienes tienen la posibilidad de elegir y luego mantienen el esfuerzo de pensar, por lo que las personas que viven en sociedades no democráticas se ven imposibilitadas de realizar una libre elección de la identidad, ya que aunque haya individuos que salvaguarden su propia identidad individual, ésta viene impuesta mayoritariamente por la presión del grupo, lo cual comporta la instauración de una uniformidad. En estos casos, podríamos decir que identidad individual e identidad colectiva forman un todo que se deriva de la imposición de 
la mayoría dominante. Es por ello que el mantenimiento de la propia identidad posee un componente de lucha y exige un esfuerzo crítico por defenderla.

Ahora bien, como hemos señalado anteriormente, esto no implica que el individuo pueda conformar su identidad sin tener en cuenta el entorno, ya que todos tenemos el deseo de ser aceptados, de poder identificarnos con los demás, de ser reconocidos, por lo que nos vemos obligados a negociar nuestra propia identidad con nuestro entorno hasta encontrar un punto de equilibrio entre nuestra identidad personal y la identidad colectiva, lo cual no equivale a aceptar una identidad impuesta; como bien afirman los profesores Jordán, Ortega y Mínguez $(2002,113)$ "la identidad personal es, en efecto, el propium de cada ser humano, en cuanto tal, el producto autónomo y libre que cada cual, desde la edad más temprana, ha ido construyendo a través de sus interacciones con los otros en entornos como los nuestros, cada vez más plurales". En cualquier caso, podemos constatar que cuando hablamos de interculturalismo, el tema de la identidad es crucial, ya que lo que pretendemos es lograr la libre expresión de nuestra propia identidad y el derecho a que ésta sea reconocida por los demás y no rechazada por el simple hecho de diferir de la identidad de la mayoría.

\section{4. ¿QUIÉNES SOMOS NOSOTROS?: LAS IDENTIDADES COLECTIVAS}

De la misma manera que los individuos necesitan desarrollar una identidad estable y sólida que les permita definir quiénes son ellos mismos, las colectividades necesitan establecer sus propias identidades. Son muchos los términos que se utilizan al respecto tales como identidad social, identidad cultural, identidad étnica, etc. En cualquier caso, de lo que se está hablando es de una colectividad de individuos que desarrollan sentimientos de pertenencia a un grupo determinado, con unas características definidas, lo cual les va a permitir identificarse a ellos mismos como grupo y diferenciarse de las otras colectividades.

Las definiciones que podemos encontrar sobre tales conceptos son numerosas; para Aguirre $(1997,31)$ la identidad étnica es un sistema cultural de referencia, a partir del cual una comunidad define su identidad grupal". Según Tajfel (1975, 376), la identidad social puede ser entendida como «aquella parte del autoconcepto de un individuo que deriva del conocimiento de su pertenencia a un grupo social, junto con la significación emocional y valorativa asociada a dicha pertenencia".

La cuestión será determinar cuál es el proceso de formación de tales identidades comunes. Para Kymlicka (1996), la respuesta es simple ya que tales identidades se derivarán de la historia, de la lengua y tal vez de la religión. Ahora bien, en tal exposición no se tiene en cuenta que los grupos están formados por individuos que poseen identidades propias, es decir, tal como defiende Anzieu (1986) no se toma en consideración el peso que las biografías de los individuos tienen en la construcción de los grupos, sin olvidar que tales identidades son una parte de la identidad personal, uno de los componentes del autoconcepto (Muñoz Sedano, 1997). 
Asimismo, y como expone el mismo Kymlicka, esas características son precisamente aquellas cosas que no se comparten en un Estado Multinacional.

Consideramos que esta idea expuesta por Kymlicka es fundamental en el tema del interculturalismo, ya que plantea el problema base de los conflictos interculturales. Cuando se está hablando de relaciones interculturales, en parte, nos estamos refiriendo a la lucha por el reconocimiento de las distintas identidades que coexisten dentro de un mismo espacio. Obviamente esta pluralidad de identidades implica la existencia de elementos diferenciadores como la lengua, la religión, la historia, los valores, etc. Cuando una persona llega a una nueva sociedad puede descubrir que su identidad no es aceptada, ya que en muchos casos pueden existir elementos de tales identidades que entren en conflicto con las características identificatorias de la sociedad de acogida. En tal caso, la persona recién llegada tiene dos opciones básicas; por una parte puede decidir integrarse de pleno en la sociedad receptora haciendo suya la identidad de tal sociedad para lograr el máximo grado de aceptación (asimilación), mientras que una segunda posibilidad puede ser establecer una lucha por el reconocimiento de su propia identidad, lo cual puede dar lugar al segregacionismo cuando tales identidades no son aceptadas por el grupo mayoritario. Por lo tanto, el objetivo a perseguir será la asunción por parte de las sociedades del pluralismo cultural que está presente en ellas. En este sentido Stickel (1987) estima que son necesarias cuatro condiciones para que pueda crecer dicho pluralismo:

- Existencia de diversidad cultural dentro de la sociedad.

- Interacción inter e intragrupos.

- Los grupos tienen que compartir aproximadamente las mismas oportunidades políticas, económicas y educativas.

- La sociedad debe valorar la identidad cultural de los grupos y de las personas.

En cualquier caso, para el desarrollo del interculturalismo es condición sine qua non que los distintos grupos adopten una actitud positiva hacia el reconocimiento de las identidades de los otros. Mi deseo de que reconozcan y respeten mi identidad implica también que yo respete y reconozca la identidad de los demás, actitud que está en la base de la capacidad de enriquecimiento cultural. Ahora bien, es necesario que en primer lugar tengamos clara cuál es nuestra propia identidad, ya que:

Si desconozco quién soy yo, no pura abstracción, sino existencia concreta, marcada por la raza, lengua, tradiciones y formas de vida, es decir, la cultura, no acertamos a ver cómo es posible ayudar a que los otros (educandos) descubran también quiénes son como seres humanos, que realizan, dentro de una cultura, una de las posibilidades de realización de la existencia humana (Ortega y Mínguez, 1997, 46). 
Bajo nuestro punto de vista, una fuerte identificación colectiva por parte de la sociedad receptora puede resultar positiva al actuar como atenuante ante la aparición de los sentimientos de pérdida de la propia identidad, ya que dicho temor "Contribuye a reaccionar de forma que, cuando no opera la clara exclusión, predominen las estrategias de larvada aculturación o absorción de los extraños" (Jordán, Ortega y Mínguez, 2002, 103). Dicha estabilidad de la identidad (colectiva en este caso) servirá de fundamento para afrontar el contacto intercultural como una fuente de enriquecimiento mutuo y para evitar el temor ante la denominada "invasión cultural. Hemos de ser conscientes de que las identidades no son estáticas, sino que están en constante estado de evolución y que pueden ser enriquecidas con las aportaciones que desde otras culturas se reciban. Tal y como afirma Aguirre (1997) las culturas no tienen membranas impermeables, al contrario, necesitan para su vitalidad el contacto con las otras culturas, recibiendo de ellas, mediante una asimilación selectiva, elementos dinamizadores (comunicación intercultural). Por otra parte, para mantener dentro de una misma sociedad un proceso de diálogo intercultural, se hace preciso un mínimo de cohesión, es decir, un esfuerzo por conocerse, escucharse y comprenderse, sin el cual no hay deliberación común, porque si un grupo considera que no es escuchado por los demás o que su punto de vista no es tenido en cuenta, se cuestiona la legitimidad democrática del grupo. Como expone Esteve (1992), la estabilidad no puede asegurarse más que cuando la legitimidad parte de un compromiso común, a partir de una deliberación común en la que es tenida en cuenta la opinión y el punto de vista de cada uno de los subgrupos. En caso contrario, puede a parecer la fragmentación y la disolución de los lazos que unen a los distintos grupos. Para hacer frente a esta situación, una educación pluralista debería basarse en el respeto a la identidad de los distintos grupos culturales "sin aceptar las visiones provincianas que pretenden fijar la identidad de un grupo humano congelando su historia en un momento determinado, favorable al grupo de presión que exhibe la foto fija" (Esteve, 2004, 101).

El propio concepto de cultura puede proporcionarnos claras pistas para entender mejor la problemática que estamos tratando:

La cultura consiste en formas de comportamiento, explícitas o implícitas, adquiridas y transmitidas mediante símbolos, y constituye el patrimonio singularizador de los grupos humanos, incluida su plasmación en objetos; el núcleo esencial de la cultura son las ideas tradicionales (es decir, históricamente generadas y seleccionadas), y, especialmente, los valores vinculados a ellas (Kroeber y Kluckhon, 1952, 181).

Pues bien, si nos centramos en dicha definición podemos llegar a entender más claramente el porqué de la resistencia de ciertos grupos ante el miedo a lo que se ha venido a denominar "invasión cultural", es decir, el temor a la convivencia con valores o personas de diferentes culturas. Para estas personas, teniendo en cuenta que la cultura propia constituye el "patrimonio singularizador", la presencia de elementos culturales ajenos puede ser vista como un atentado contra la propia cultura ya que podría traducirse en un posible debilitamiento de la identidad del grupo. 
Por otra parte y dado que la cultura es uno de los elementos fundamentales constituyentes de cualquier sociedad, esta presencia extranjera podría derivar en una pérdida de consistencia en la cohesión social. Ahora bien, cabría preguntarse si esto sucede con los grupos cuya identidad es sólida y estable. Parece demostrado que las sociedades o los grupos tradicionalmente en minoría rechazan en mayor medida la influencia cultural externa, algo que podría responder a un mecanismo de defensa motivado por la imposición histórica de una cultura ajena y consecuentemente a la instauración obligada de una identidad cultural que no dejaba de ser ficticia. El problema es que, en la actualidad, los grupos sociales que se niegan al contacto intercultural están abocados al aislamiento, lo cual se traduce en una decadencia de esas sociedades. Fenómenos como la creciente inmigración, la libre circulación de ciudadanos dentro de la Unión Europea o la expansión de la información y la comunicación a través de las nuevas tecnologías hace que hoy más que nunca se hable de la denominada "aldea global" donde el contacto intercultural es una característica configuradora. Permanecer de espaldas ante esta situación supone simplemente negar la realidad.

\section{El DOCENTE ANTE LA IDENTIDAD DE SUS ALUMNOS}

A raíz de la importancia del tema de la identidad a la hora de abordar la educación en contextos de diversidad cultural decidimos preguntar a los profesores acerca de la identidad de sus alumnos. Dicha cuestión se enmarca en un estudio llevado a cabo en el Departamento de Teoría e Historia de la Educación de la Universidad de Málaga con una muestra de 87 profesores de primer ciclo de Educación Secundaria Obligatoria, pertenecientes a 17 centros educativos de la zona de Campo de Gibraltar (La Línea de la Concepción, San Roque y Algeciras). A través de un cuestionario elaborado a tal efecto y de entrevistas personales sondeamos las opiniones de los docentes y directores de los centros de dicha zona sobre múltiples cuestiones relacionadas con la atención al alumnado en un contexto de educación intercultural. Una de esas cuestiones iba dirigida a conocer concretamente la valoración que realizaban los docentes acerca de las preferencias de sus alumnos respecto a sus identidades.

Como puede observarse en la tabla 1, dividimos la cuestión entre alumnos inmigrantes y alumnos gitanos, ya que dichos grupos culturales son los más significativos en la zona de estudio, atendiendo a sus edades (recordemos que la consolidación de la identidad se produce tras el periodo de la adolescencia). Tal división por edades, nos ayudará a conocer la opinión del profesorado sobre las distinciones que respecto a la identidad pueden producirse en sus alumnos en función de la cultura y del momento de desarrollo. Ofrecimos a los profesores tres opciones de respuesta (Merino, 2003):

a) Que, según su opinión, el deseo de sus alumnos fuera integrarse en la cultura mayoritaria manteniendo su identidad. 
EL RESPETO A LA IDENTIDAD COMO FUNDAMENTO DE LA EDUCACIÓN INTERCULTURAL

b) Que, bajo su punto de vista, sus alumnos desearan integrarse en la cultura mayoritaria aunque esto supusiera la renuncia a su identidad.

c) Que, a su entender, sus alumnos primaran el mantenimiento de su identidad aunque ello les comportara problemas de integración en la cultura mayoritaria.

Veamos los resultados obtenidos a tal efecto:

TABLA 1

VALORACIÓN POR PARTE DE LOS PROFESORES ACERCA DE LAS PREFERENCIAS

DE LOS ALUMNOS RESPECTO A SUS IDENTIDADES

(EN FUNCIÓN DEL GRUPO Y DEL MOMENTO DE DESARROLLO)

$$
\mathrm{N}=87
$$

\begin{tabular}{|l|c|c|c|}
\hline & $\begin{array}{c}\text { Integrarse en la } \\
\text { cultura mayoritaria } \\
\text { manteniendo } \\
\text { su identidad }\end{array}$ & $\begin{array}{c}\text { Integrarse en la } \\
\text { cultura mayoritaria } \\
\text { renunciando } \\
\text { a su identidad }\end{array}$ & $\begin{array}{c}\text { Mantener } \\
\text { su propia } \\
\text { identidad }\end{array}$ \\
\hline $\begin{array}{l}\text { Inmigrantes llegados } \\
\text { de pequeños }\end{array}$ & 55 & 5 & 0 \\
\hline Niños gitanos & $91,7 \%$ & $8,3 \%$ & $0 \%$ \\
\hline Inmigrantes llegados & 44 & 1 & 37 \\
de mayores & $53,7 \%$ & $1,2 \%$ & $45,1 \%$ \\
\hline $\begin{array}{l}\text { Adolescentes } \\
\text { gitanos }\end{array}$ & $72 \%$ & $1,7 \%$ & 17 \\
\hline
\end{tabular}

La representación gráfica de los resultados nos ayudará a apreciar con mayor claridad las diferencias obtenidas: 


\section{GRÁFICO 1}

VALORACIÓN POR PARTE DE LOS PROFESORES ACERCA DE LAS PREFERENCIAS

DE LOS ALUMNOS RESPECTO A SUS IDENTIDADES

(EN FUNCIÓN DEL GRUPO Y DEL MOMENTO DE DESARROLLO)

$$
\mathrm{N}=87
$$

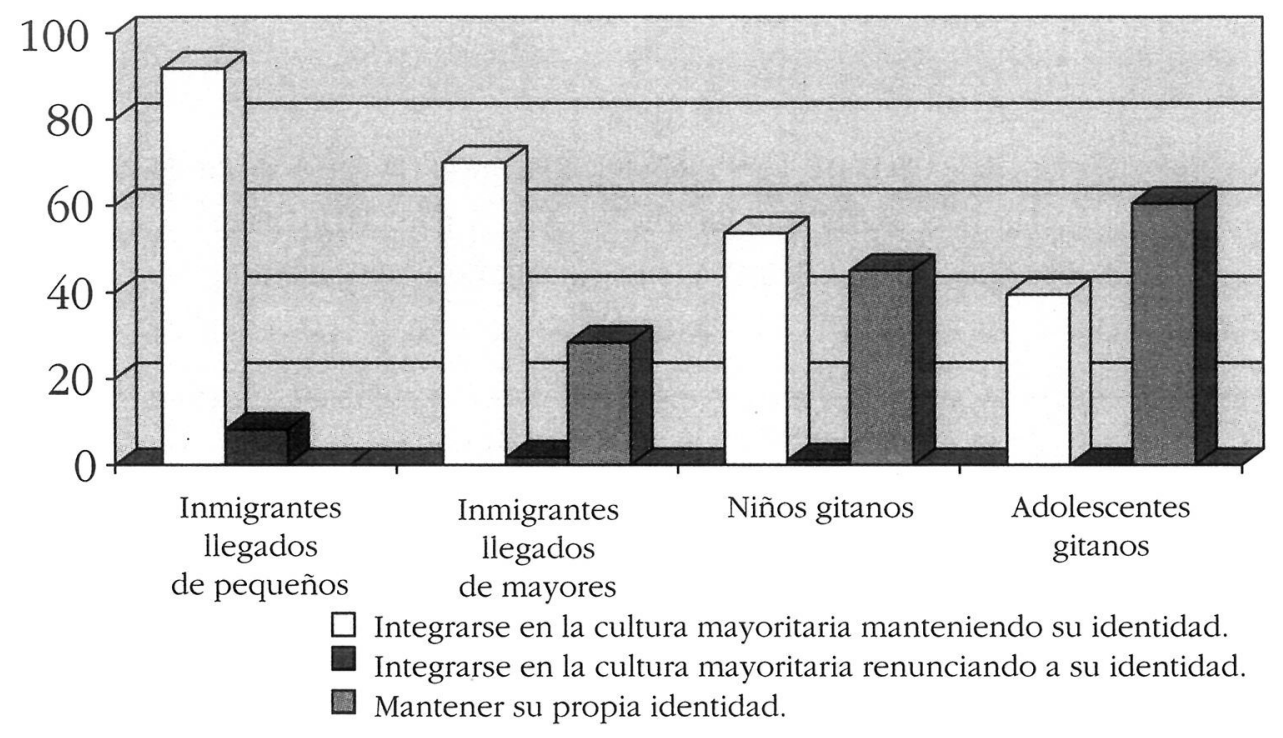

Efectivamente, podemos comprobar que, según el profesorado encuestado, el grupo que en mayor medida desea integrarse en la cultura mayoritaria manteniendo su propia identidad es el formado por los inmigrantes llegados de pequeños. Los datos reflejan una muy baja proporción en lo que respecta a la renuncia de la propia identidad en todos los grupos culturales. Asimismo, resulta muy significativo que el colectivo de alumnos gitanos adolescentes alcance una proporción del $60,5 \%$ en lo que respecta al mantenimiento de su propia identidad por encima de su integración, frente al $28,3 \%$ de los inmigrantes llegados mayores. Por tanto, el grupo formado por los adolescentes gitanos es el único que, según los docentes encuestados, prefiere mantener su propia identidad por encima de su integración, frente al mantenimiento de ésta integrándose en la cultura mayoritaria.

Una vez que procedimos a la realización de las entrevistas, preguntamos a los docentes acerca de las repercusiones educativas que podría tener dicha realidad. Algunas respuestas resultan muy interesantes:

Pues que no asumen otro... vamos, que no asumen las diferencias que puede haber con otras personas. Una persona que está más abierta puede aceptar mejor los consejos o los valores o aquello que sea bueno de un sitio u otro y que no tienen por qué anular su propia cultura (Profesor nํㅜ 16. Algeciras).

Respecto al hecho de que los adolescentes gitanos intenten mantener su propia identidad en mayor medida que los alumnos más pequeños, una directora realiza la siguiente reflexión: 
EL RESPETO A LA IDENTIDAD COMO FUNDAMENTO DE LA EDUCACIÓN INTERCULTURAL

Bueno, los niños pequeños en primaria aceptan lo que su profesor les diga, pero los mayores ya es otra cosa; de todas maneras te digo que aunque haya mucha diversidad en las clases, tampoco se les da la oportunidad de expresarse, porque, a ver, ¿cuándo se les dice a estos niños que nos hablen de sus costumbres...?, porque las vemos como raras y horribles... pero claro, el niño a partir de los doce años entra en la adolescencia, empieza a cuestionarse las cosas y no acepta todo lo que diga el profesor, todo lo contrario... (Directora no 3. La Línea de la Concepción).

Efectivamente, y como hemos defendido a lo largo de este artículo, el alumno necesita sentirse valorado, en primer lugar atendiendo a "quién es él" (identidad individual) y en segundo lugar respetando los valores y costumbres de su cultura (identidad colectiva). La presencia en el centro educativo, en el aula, de elementos que los alumnos de culturas minoritarias identifiquen como propios de su cultura contribuirá de manera decisiva a que no perciban la institución escolar como algo lejano, sino como un entorno en el que su identidad es respetada y valorada, pero siempre teniendo en cuenta que "tanto los profesores como los alumnos autóctonos deberían ver a un argelino, un pakistaní o un chino no como ejemplares de las culturas originarias respectivas, sino como personas concretas que tienen, también y a veces de modo psicológicamente marcado, ciertos códigos culturales influyentes en su forma de pesar y actuar" (Jordán, Ortega y Mínguez, 2002, 115).

Por tanto, podemos comprobar que los alumnos desean mayoritariamente integrarse en el entorno cultural en el que coexisten, pero a su vez reclaman que dicha integración no suponga óbice para que sean valorados por quiénes son ellos mismos.

\section{CONCLUSIONES}

El fin de la educación intercultural es lograr la convivencia, el respeto y la valoración mutua entre los alumnos para que ese clima de entendimiento y de tolerancia se traslade a la sociedad en la que vivimos. A pesar de ello, sabemos que uno de los problemas que repetidamente se ha manifestado a la hora de establecer relaciones y vínculos interculturales ha sido la relevancia que hemos otorgado a las diferencias en detrimento de los aspectos que nos unen, que nos igualan, y en la base de dichos aspectos hay uno fundamental: todos somos personas. Precisamente ése debe ser el fundamento de la educación intercultural, es decir, para que ese deseo de respeto y entendimiento pueda transformarse en una realidad será necesario que todos los alumnos, que todas las personas se sientan valoradas, apreciadas y aceptadas por quiénes son y no únicamente por su procedencia y su cultura de origen. Como defiende el profesor Bernal:

Aspiramos a una escuela - y a un mundo- donde aquellos que protagonizan la educación sean acogidos como personas, como seres dotados de dignidad y de libertad. Un lugar, un espacio, donde cada sujeto tenga su reconocimiento, su valor, su propia voz. Pues bien, la respuesta pedagógica al problema de la integración de 
inmigrantes pasa por la promoción adecuada de los procesos de construcción de las identidades personales, de la identidad personal de cada cual (Bernal, 2002, 3).

La escuela es uno de los principales escenarios en el que los sujetos construyen sus identidades en interacción con los demás; la promoción adecuada de valores como la igualdad, el respeto, la solidaridad, el pluralismo, la tolerancia, hacia los otros como individuos, como personas y no sólo hacia las "otras culturas", hacia "las otras tradiciones" favorecerá el adecuado desarrollo de unas identidades personales respetuosas con los demás y capaces de reconocer y aceptar positivamente las diferencias. Éste será, a nuestro entender, el primer paso, la "primera piedra" a colocar en el complejo desarrollo de la educación intercultural: el reconocimiento de esa alteridad del otro, como paso previo al reconocimiento de su "bagaje cultural" como uno de los elementos configuradores de ese individuo.

El riesgo de centrar los procesos de educación intercultural únicamente en la aceptación y el respeto de las culturas presentes en el contexto educativo estriba, a nuestro entender, en la posible "cosificación" del sujeto en tanto que miembro de una cultura determinada con unas características específicas que, en muchos de los casos, le hemos atribuido nosotros mismos. El problema reside en que la identidad de un sujeto no tiene por qué estar formada únicamente por las influencias de una cultura o de un grupo determinado, es decir, que "la posibilidad de pertenencia de un individuo a más de un grupo cultural simultáneamente y de participar en más de una subcultura, de normas y referencias no necesariamente coherentes entre sí, multiplica los entrecruzamientos de identidades e impide la identificación automática de individuo con grupo cultural" (Ortega, 2004, 60).

A lo largo de este artículo hemos argumentado las inequívocas relaciones existentes entre identidad individual e identidad colectiva, y es por ello precisamente por lo que defendemos que un adecuado desarrollo de la identidad personal basado en la promoción de valores positivos será una puerta abierta al reconocimiento de "la/s cultura/s del otro": si somos capaces de entender, respetar y apreciar a la otra persona, consecuentemente estaremos favoreciendo la valoración de sus características sociales y culturales ya que éstas formarán parte de su propia individualidad. Centrarnos exclusivamente en la identidad colectiva de los individuos supone un ejercicio reduccionista y ficticio al presuponer que todos los sujetos comparten unas características definidas. No nos equivoquemos, no se trata de negar el valor de los grupos, de las culturas, sino de atribuir un mayor protagonismo al individuo que, desde la libertad que comporta el respeto a su individualidad, podrá elegir autónomamente su propia vía de autorrealización. 
EL RESPETO A LA IDENTIDAD COMO FUNDAMENTO DE LA EDUCACIÓN INTERCULTURAL

BiBLIOGRAFÍA

Aguirre, A. (1997) Cultura e identidad cultural. Introducción a la Antropología. Barcelona, Bárdenas.

ANZIEU, D. (1986) El grupo y el inconsciente. El imaginario grupal. Madrid, Ed. Biblioteca Nueva.

Bernal, A. (2002) La construcción de la identidad personal en el currículum escolar. A propósito de la integración de las poblaciones de inmigrados, desde el enfoque del pluralismo. Addenda presentada al XXI Seminario Interuniversitario de Teoría de la Educación. Granada.

CASTILlo, S. (2004) Hacia una evaluación facilitadora de la identidad, en AA.VV. La educación en contextos multiculturales: diversidad e identidad. Valencia, Universitat de València, 377-411.

ERIKSON, E. (1989) Identidad: juventud y crisis. Madrid, Taurus Humanidades.

Esteve, J. M. (1992) Multicultural education in Spain: The Autonomus Communities faces the challenge of european unity, Educational Review, 44 (3), 255-272.

- (2004) La formación del profesorado para una educación intercultural, Bordón, 56 (1), 95-115.

Esteve, J. M.; Merino, D.; Rius, F.; Canto, B. y Ruiz, C. (2003) Autoconcepto y respuestas agresivas en un contexto de educación intercultural, Revista de Educación, 332, 357-381.

JordÁn, J. A.; ORTEGA, P. y Mínguez, R. (2002) Educación intercultural y sociedad plural, Teoría de la Educación. Revista Interuniversitaria, 14, 93-119.

Froufe, S. (1999) Educación intercultural y pedagogía de la interculturalidad, Pedagogía Social. Revista Interuniversitaria, Monográfico Educación Intercultural-1, 9-26.

KIRSCHNER, D. (1992) Understanding adoptes who kill: Dissociation, patricide and the psychodynamics of adoption, International Journal of Offender Therapy and Comparative Criminology, 36, 323-333.

Kroeber, A. L. y KluCKHohn, C. (1952) Culture, a critical review of concepts and definitions, Papers of the Peabody Museum (Harvard University), 47, ํㅡ 1.

KymLicKA, W. (1996) Ciudadania multicultural. Barcelona, Paidós.

LONG, D. E. (1990) The anatomy of terrorism. New York, Free Press.

MAALouf, A. (1999) Identidades asesinas. Madrid, Alianza Editorial.

Malewska-Peyre, H. (1982) Crise d'identité et problèmes de déviance chez les jeunes immigrés. Vaucresson, CRIV.

MERINO, D. (2003) Concepciones de los profesores y autoconcepto y agresividad de los alumnos en un contexto de educación intercultural. Málaga, Servicio de Publicaciones de la Universidad de Málaga.

Monreal, P.; MuÑoz, J. y MarTínez, M. (1990) Pertenencia grupal en la adolescencia. Comunicación presentada al III Congreso Nacional de Psicología Social. Santiago de Compostela.

MuÑoz SedAno, A. (1997) Educación intercultural. Teoria y práctica. Madrid, Escuela Española.

ORTEGA, P. (2004) Cultura, valores y educación: principios de integración, en AA. VV. La educación en contextos multiculturales: diversidad e identidad. València, Universitat de València, 48-80.

Ortega, P. y Mínguez, R. (1997) El reto de la educación intercultural, Teoría de la Educación, Revista Interuniversitaria, 9, 41-53. 
EL RESPETO A LA IDENTIDAD COMO FUNDAMENTO DE LA EDUCACIÓN INTERCULTURAL

SCHOENFELD, C. G. (1988) Blacks and violent crime: A psychoanalytically oriented analysis. Journal of Psychiatry and Law, 16, 269-301.

SORIANO, E. (2004) La construcción de la identidad cultural en contextos multiculturales, en AA.VV. La educación en contextos multiculturales: diversidad e identidad. Valéncia, Universitat de València, 187-218.

STICKEL, G. W. (1987) Cultural Pluralism and the Schools: Theoretical Implications for the Promotion of Cultural Pluralism. Trabajo presentado a la Annual Conference of the American Association of Colleges for Teacher Education. Washington, D.C.

TAJFeL, H. (1975) La categorización social, en MoscovicI, S. (ed.). Introducción a la Psicología Social. Barcelona, Planeta, 351-387.

TAYLOR, C. (1996) Identidad y reconocimiento, Revista Internacional de Filosofía Política, 6 , 10-19.

WieHE, V. R. (1991) Perilous rivalry. Lexington, Heath-Lexington Books. 